\title{
Correction to: Characteristics of membranoproliferative glomerulonephritis based on a new classification at a single center
}

\author{
Marie Nakano $^{1} \cdot$ Kazunori Karasawa $^{1} \cdot$ Takahito Moriyama $^{1} \cdot$ Keiko Uchida $^{1} \cdot$ Kosaku Nitta $^{1}$
}

Published online: 3 September 2019

(c) Japanese Society of Nephrology 2019

\section{Correction to: Clinical and Experimental Nephrology} https://doi.org/10.1007/s10157-019-01716-7

In the Original publication, the authors found few errors in the text. The corrections are given below:

1. The "Complement (CP)-mediated MPGN" should read as "Alternative pathway (AP)-mediated MPGN" throughout the article.

2. Under the Conclusion and Discussion section, the sentence "These results suggested no significant differences in complement activation of the solid phase in local glomeruli and therefore no equivalence in renal prognosis" should read as "These results suggested no significant differences in complement activation of the solid phase in local glomeruli and therefore equivalent in renal prognosis".

3. Under the Statistical analysis, the text "Categorical variables were expressed as the mean \pm standard deviation unless specified otherwise and were compared using the unpaired $t$ test or Mann-Whitney $U$ test" should read as "Categorical variables were expressed as the number and percentage and were compared using Fisher's exact test" to analyze differences in laboratory and histological data.

Publisher's Note Springer Nature remains neutral with regard to jurisdictional claims in published maps and institutional affiliations.

The original article can be found online at https://doi.org/10.1007/ s10157-019-01716-7.

Kazunori Karasawa

ichitoku@hotmail.co.jp

Department of Medicine, Kidney Center, Tokyo Women's

Medical University, 8-1 Kawada-Cho, Shinjuku-ku,

Tokyo 162-8666, Japan 